( $u_{-}=$Elektronendriftgeschwindigkeit). Der entsprechende induzierte Stromimpuls folgt aus (A.1) mit (A.9). Wir berechnen den Stromimpuls zur Zeit $t$, welchen eine bei $t=0$ gestartete Lawine erzeugt; sie befindet sich am Ort $x=u_{-} t$ und besteht aus $\left(q_{0} / e\right) e^{t / \tau}$ Elektronen. Es wird

$$
i(t)=\frac{q_{0}}{D \tau} \int_{0}^{t} \dot{x}\left(t, t_{\mathrm{s}}\right) e^{t_{\mathrm{s}} / \tau} \mathrm{d} t_{\mathrm{s}}+\frac{q_{0} \dot{x}(0)}{D}
$$

mit $\dot{x}\left(t, t_{\mathrm{s}}\right)$ aus (A.9). Der letzte Summand berücksichtigt die beim Lawinenstart bereits vorhandenen Primärelektronen. Bei der Ausführung der Integration (A.10) erhält man einen Ausdruck $\sim U_{\sim} \cos \omega t$, welcher im Fall $v \gg 1 / \tau$ und bei gleichzeitigem Übergang auf Zeigergrößen $\tilde{U}=U_{\sim} e^{j \omega t}$ die folgende einfache Form annimmt:

$$
\tilde{i}=\tilde{U} \frac{e q_{0} e^{t / \tau}(v-j \omega)}{m D^{2}\left(v^{2}+\omega^{2}\right)}=\tilde{U} \cdot \mathscr{Y}_{1} .
$$

$\mathfrak{V}_{1}$ ist aber der komplexe Leitwert des Lawinenraums nach Abb. 4 bei Anwesenheit nur einer einzigen Lawine der Ladung $q_{0} e^{t / \tau}$. Bei genügend vielen, im Mittel gleichmäßig verteilten Lawinen der mittleren Häufigkeit $z_{\text {ges }}$ Lawinen/sec und der Laufzeit $t_{1}$ findet man mit $I=z_{\text {ges }} q_{0} e^{\alpha D}$ leicht den Leitwert der gesamten Strecke aus

$$
\begin{gathered}
\mathfrak{Y}=\frac{I \cdot t_{1}}{q_{0} e^{\alpha D} \cdot D} \int_{0}^{D} \mathfrak{Y}_{1} \mathrm{~d} x \\
\mathrm{zu} \quad \mathfrak{Y}=\frac{e I(v-j \omega)}{\alpha D m u_{-} D\left(\omega^{2}+\nu^{2}\right)} \cdot \frac{e^{\alpha D}-1}{e^{\alpha D}} \\
\approx \frac{e I(v-j \omega)}{\alpha D m u_{-} D\left(\omega^{2}+v^{2}\right)} .
\end{gathered}
$$

\title{
Some Decomposition Routes in the Mass Spectra of Aromatic Carboxylic Acids
}

\author{
J. H. Beynon, B. E. Job, and A. E. Williams \\ Imperial Chemical Industries Limited, \\ Dyestuffs Division and Petrochemical and Polymer Laboratory, \\ Hexagon House, Blackley, Manchester 9
}

(Z. Naturforschg. 20 a, 883-887 [1965] ; received 21 May 1965)

\begin{abstract}
The mass spectra of benzoic acid, phthalic acid, isophthalic acid and terephthalic acid, together with the analogues deuterated on the carboxylic acid group have been studied. Exchange of the deuterium atom with hydrogen atoms on the positions ortho to a carboxylic acid group on the aromatic ring has been studied using meta-stable peaks.
\end{abstract}

\section{Experimental}

The mass spectra of benzoic acid, phthalic acid, isophthalic acid and terephthalic acid, together with acids in which the acid protons were replaced by deuterium have been studied in an A.E.I. type M.S. 9 doublefocussing mass spectrometer ${ }^{1}$. The phthalic acid is too thermally unstable to enable its spectrum to be obtained via a capillary leak from a heated gas inlet system $^{2}$. Therefore, all spectra were obtained using the direct inlet method ${ }^{3-5}$; for the dibasic acids the sample requires to be heated to a temperature of only about $100{ }^{\circ} \mathrm{C}$ to enable its spectrum to be obtained. Decomposition of phthalic acid into phthalic anhydride is not observed to any great extent during the time of measurement below a temperature of about $125^{\circ} \mathrm{C}$.

All the deuterated samples were prepared by direct exchange with an excess of deuterium oxide (99.7\%).

1 R. D. Craig, B. N. Green, and J. D. Waldron, Chimia 17, 33 [1963].

2 F. W. McLafferty and R. S. Gohlke, Anal. Chem. 31, 2076 [1959].

3 R. I. ReEd, J. Chem. Soc. 1958, 3432.
The acids were purified by re-crystallising from hot deuterium oxide. Introduction of the deuterated samples to the mass spectrometer, however, resulted in a partial reverse exchange due to residual water in the instrument. The final deuteration was therefore achieved in situ in the mass spectrometer by the simultaneous introduction of deuterium oxide and the partially deuterated acid sample in the spectrometer source. The degree of exchange which had been achieved was monitored using the molecular ions in the mass spectra. The fact that the samples were not exclusively deuteromaterial was not normally a serious disadvantage as the meta-stable peaks used in the analysis do not depend on the state of purity of the sample. When necessary (as mentioned below for the isophthalic and terephthalic acid spectra) correction of the spectrum was made by substraction from it of the appropriately normalized spectrum of the undeuterated material.

4 K. Biemann, 10th Annual Meeting Committee E-14 ASTM, New Orleans, La., 1962.

5 J. H. Beynon, R. A. Saunders, and A. E. Williams, Appl. Spectr. 17, 63 [1963]. 
Many meta-stable transitions were observed in all the mass spectra; in all the diagrams shown below, which discuss some of the decomposition paths, all fragmentation steps in which a meta-stable peak was observed are indicated. Where no indication is given of a meta-stable peak it was not, in fact, observed. The symbolism used is that proposed by Djerassi ${ }^{6}$ and recently recommended by SHANNon ${ }^{7}$.

\section{Results}

The major decomposition processes occurring in benzoic acid are shown in Fig. 1. The molecular ion of mass 122 decomposes along two paths. Along the first a hydroxyl group is lost giving rise to a metastable peak at 90.4 , and the daughter ion of massto-charge ratio 105 then loses carbon monoxide (shown by a meta-stable peak at 56.5) to give the ion $\mathrm{C}_{6} \mathrm{H}_{5}^{+}$of mass 77 ; this further fragments by loss of $\mathrm{C}_{2} \mathrm{H}_{2}$ to produce the ion of mass 51 (metastable peak at 33.8 ). The other decomposition path occurs with much lower probability. Carbon monoxide is lost (meta-stable peak at 72.4), followed by the further loss of $\mathrm{CO}$ or $\cdot \mathrm{CHO}$ to give the ions of mass 66 and 65 respectively; no meta-stable peaks are apparent for these transitions.

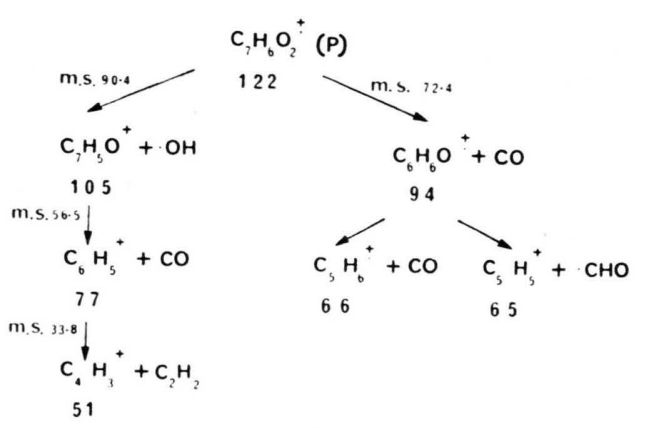

Fig. 1. Major decomposition paths in the mass spectrum of benzoic acid.

Benzoic acid- $\mathrm{d}_{1}$ shows the analogous decomposition routes as shown in Fig. 2. However, loss of $\cdot \mathrm{OH}$ from the parent ion is observed in addition to the expected loss of $\mathrm{OD}$ in the first decomposition route. The corresponding meta-stable peaks are observed

(a) $\mathrm{C}_{7} \mathrm{H}_{5} \mathrm{DO}_{2} \cdot{ }^{+}$(parent) $\rightarrow \mathrm{C}_{7} \mathrm{H}_{5} \mathrm{O}^{+}+\cdot \mathrm{OD}$ (m. s. 89.6)

${ }^{6}$ H. Budzikiewicz, C. Djerassi, and D. H. Williams, Interpretation of Mass Spectra of Organic Compounds, Holden-Day, San Francisco 1964, pp. xi, xii. (b) $\mathrm{C}_{7} \mathrm{H}_{5} \mathrm{DO}_{2} \cdot{ }^{+}$(parent) $\rightarrow \mathrm{C}_{7} \mathrm{H}_{4} \mathrm{DO}^{+}+\cdot \mathrm{OH}$

(m.s. 91.3)

The relative probability of the two reaction paths involving loss of $\cdot \mathrm{OD}$ or $\cdot \mathrm{OH}$ can be judged from the heights of the meta-stable peaks which accompany them. Fig. 3(a) illustrates that the peak at 91.3 is twice as large as that at 89.6. This would be expected if, before decomposition, the deuterium could equilibrate with the two hydrogen atoms ortho to the carboxylic acid group on the benzene ring.

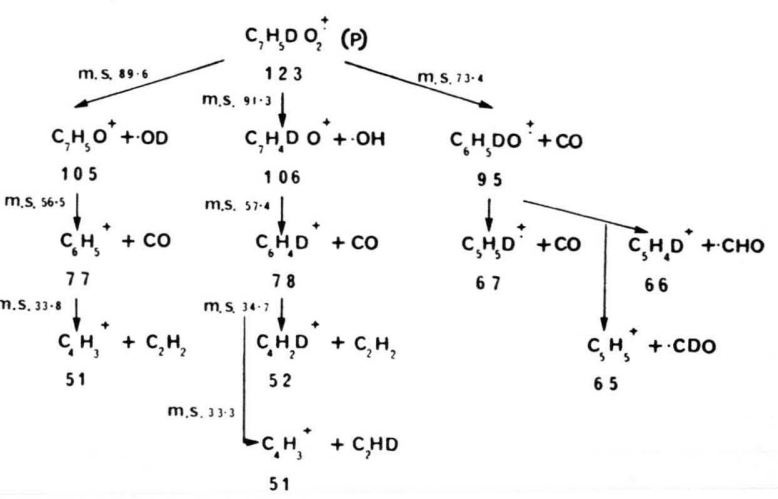

Fig. 2. Major decomposition paths in the mass spectrum of benzoic acid- $\mathrm{d}_{1}$.

If the positive charge on the molecular ion of benzoic acid is located almost entirely on the oxygen atoms of the carboxylic group due to loss of one of the oxygen lone pair electrons, it is possible to visualise the accommodation of an extra hydrogen atom from one of the ortho positions on the ring, by way of an intermediate species such as<smiles>O=C(O)c1ccccc1</smiles>

Structure [A]

in which the charge resonates between the two oxygens. When the side-chain is deuterated, and if the single deuterium atom and two hydrogen atoms can exchange freely via such an intermediate before decomposition, one expects a ratio of $2: 1$ for the probability of loss of $\cdot \mathrm{OH}$ or $\cdot \mathrm{OD}$, as found for the ratio of the accompanying meta-stable peaks. For the meta-stable peak to be detected, the ion must have a half-life of the order $10^{-5}$ secs. This is, of course,

7 J. S. Shannon, Proc. Roy. Australian Chem. Inst. 31, 323 [1964]. 
much longer than the period of rotation of the carboxylic acid group.

The peaks at masses 106 and 105 are not in this ratio, that at mass 105 being much the larger; even after correction for the presence of undeuterated material this remains the case. This shows that the greater part of the 105 peak arises by loss of $\cdot \mathrm{OD}$ in a much faster decomposition, not accompanied by equilibration. Meta-stable peaks at masses 57.4 and 56.5 which arise by further decomposition are of the same approximate ratio as the ions of masses 106 and 105 from which they arise.

(a)

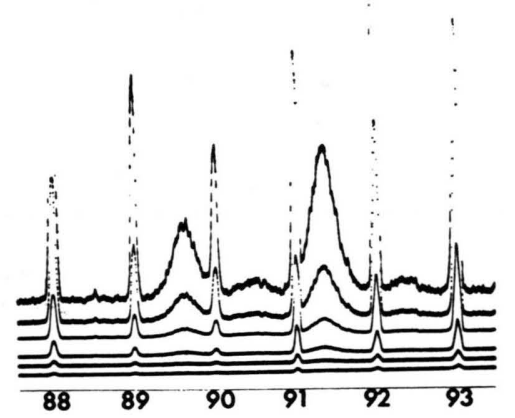

(b)

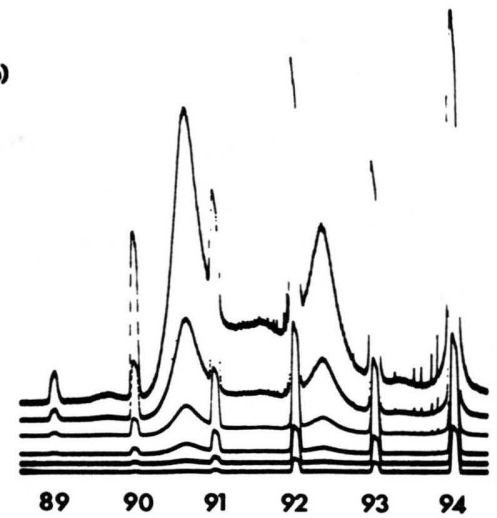

Fig. 3. (a) Meta-stable peaks resulting from loss of $\cdot \mathrm{OD}$ and - $\mathrm{OH}$ from the molecular ion in the mass spectrum of benzoic acid- $\mathrm{d}_{1}$. (b) Meta-stable peaks resulting from loss of -OD and $\cdot \mathrm{OH}$ from the ion of mass-to-charge ratio 124 in the mass spectrum of phthalic acid- $\mathrm{d}_{2}$.

The reaction paths in the case of phthalic acid are shown in Fig. 4. It is seen that a major frag. mentation path involves the loss of $\mathrm{CO}_{2}$ from the molecular ion (meta-stable peak at 89.7) to produce the ion $\mathrm{C}_{7} \mathrm{H}_{6} \mathrm{O}_{2} \cdot{ }^{+}$which has the same elemental compositions as the benzoic acid molecular ion. Sub- sequent decomposition along this route to produce the ions of mass 105,77 and 51 is the same as that described for benzoic acid itself. The alternative route involves the loss of $\cdot \mathrm{OH}$ followed by the loss of $\mathrm{CO}$ (meta-stable peak at 98.2) to produce the ion $\mathrm{C}_{7} \mathrm{H}_{5} \mathrm{O}_{2}{ }^{+}$; loss of successive molecules of $\mathrm{CO}$ results in the ions of mass 93 and 65 (meta-stable peaks at 71.5 and 45.4 respectively).

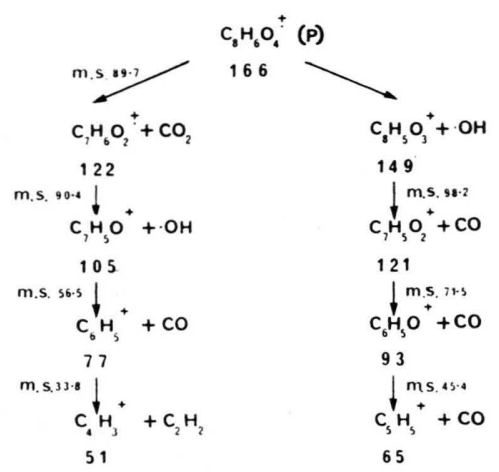

Fig. 4. Major decomposition paths in the mass spectrum of phthalic acid.

Phthalic acid- $\mathrm{d}_{2}$ follows the fragmentation routes that would be predicted from the spectrum of the natural species; the reaction paths are shown in Fig. 5. Two features of its spectrum deserve comment, however. Firstly, the loss of -OD from the parent ion is not accompanied by a meta-stable peak, and there is no exchange of the acid deuterium with ring hydrogen atoms; no loss of $\cdot \mathrm{OH}$ is observed therefore. This indicates that equilibration of the acid deuterium atoms with the hydrogen atoms ortho to the carboxylic acid group does not occur. This is probably a consequence of the steric configuration of the carboxylic acid groups, which restricts their rotation about the bonds joining these groups to the ring. Secondly, the loss of $\mathrm{CO}_{2}$ from the parent ion (meta-stable peak at 91.5) produces the ion $\mathrm{C}_{7} \mathrm{H}_{4} \mathrm{D}_{2} \mathrm{O}_{2}{ }^{+}$; rotation of the carboxylic acid group is no longer hindered, and equilibration of the hydrogen and deuterium atoms is possible. Loss of both $\cdot \mathrm{OD}$ and $\cdot \mathrm{OH}$ is observed (meta-stable peaks at 90.6 and 92.3 respectively) and since on this occasion there are two deuterium atoms and one hydrogen atom involved in the equilibration the expected ratio of these meta-stable peaks is $2: 1$. The observed ratio is close to this value [see Fig. $3(\mathrm{~b})$ ], but measurement of the peaks is complicated by their partial overlap with the meta-stable peak at $91.5\left(168^{+} \rightarrow 124^{+}+\mathrm{CO}_{2}\right)$ which is an example of 
the wide meta-stable peaks sometimes observed when a stable neutral species is removed ${ }^{8}$. The further fragmentations in the spectrum are directly analogous to those observed in the natural phthalic acid spectrum.

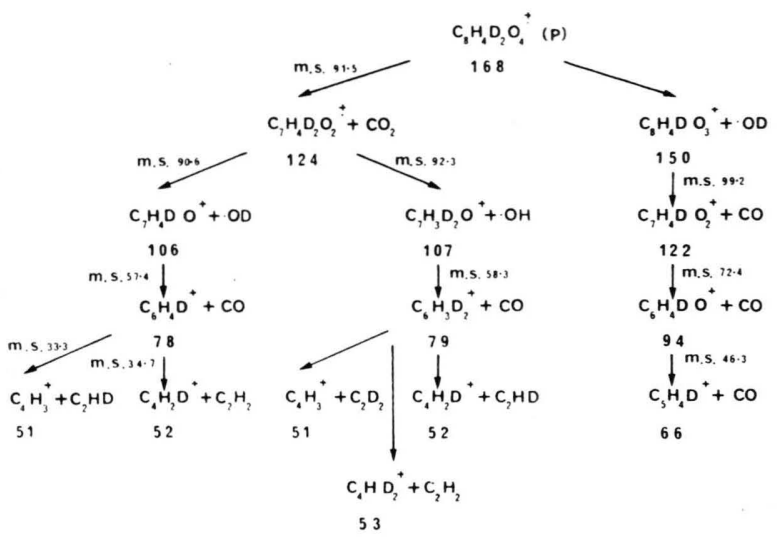

Fig. 5. Major decomposition paths in the mass spectrum of phthalic acid- $\mathrm{d}_{2}$.

Once again the large ratio of peak heights at masses 106 and 107 and the similar ratio of meta-stable peaks at masses 57.4 and 58.3 shows an alternative and much faster decomposition process. In this case, confirmation that the deuterium in the starting material is located entirely on the carboxylic acid groups can be obtained by thermal degradation of the sample to phthalic anhydride: there is no evidence from its mass spectrum of deuteration of this degradation product.

The mass spectra of terephthalic acid and isophthalic acid are very similar to each other but different from that of phthalic acid. The principal difference is that the fragmentation route involving the initial loss of $\mathrm{CO}_{2}$ from the parent ion is absent in the terephthalic and isophthalic acid spectra. This is believed to be because the carboxylic acid groups in these molecules are separated on the benzene ring. The analogous intermediate species to<smiles>O=C(O)c1ccccc1C(=O)O</smiles>

Structure [B]

which can be visualised in phthalic acid, cannot be formed under these structural conditions.

\footnotetext{
8 J. H. Beynon, R. A. Saunders, and A. E. Williams, Z. Natur-
} forschg. 20 a, 180 [1965].
The decomposition path involving the initial loss of $\cdot \mathrm{OH}$ from the parent ion is the same as that described for phthalic acid; in the case of the isoand terephthalic acids, however, the loss of the $\cdot \mathrm{OH}$ moiety is accompanied by a meta-stable peak at 133.7. The parent ion in the terephthalic and isophthalic acid spectrum can also lose $\mathrm{CO}$ to a small extent; no meta-stable peak is observed, and no important fragmentation route is initiated by this decomposition. Other peaks, characteristic of the structures are observed at mass-to-charge ratios of 74 , $75,76,77,50,51$, and $45\left(\cdot \mathrm{COOH}^{+}\right)$. However, no meta-stable peaks related to these peaks are observed so that the fragmentation routes by which they are formed cannot be stated with certainty.

The decomposition paths described are illustrated in Fig. 6.

$$
\text { m.s. }
$$

Fig. 6. Major decomposition paths in the mass spectra of terephthalic acid and isophthalic acid.

The spectra of terephthalic acid- $\mathrm{d}_{2}$ and isophthalic acid- $d_{2}$ show the same fragmentation paths (Fig. 7). In both cases the rotation of the carboxylic acid groups about the bonds joining them to the ring is possible and equilibration of the acid deuterium atoms with the ring hydrogen atoms ortho to the acid group is observed. The two deuterium atoms in terephthalic acid- $\mathrm{d}_{2}$ may exchange with four hydrogen atoms so that if equilibration is complete the meta-stable peak resulting from the process $168^{+} \rightarrow 151^{+}+\cdot \mathrm{OH} \quad$ (meta-stable peak at 135.7) would be expected to be twice as high as that resulting from the process $168^{+} \rightarrow 150^{+}+{ }^{\circ}$ OD (metastable peak at 133.9).

Similarly, in isophthalic acid- $\mathrm{d}_{2}$, the equilibration would take place between two deuterium atoms and three hydrogen atoms. The meta-stable peaks resulting from the loss of $\cdot \mathrm{OH}$ and $\cdot \mathrm{OD}$ would therefore be predicted to be in the ratio $3: 2$. 


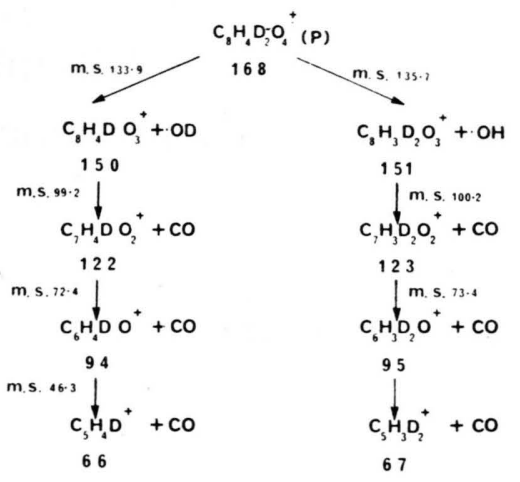

Fig. 7. Major decomposition paths in the mass spectra of terephthalic acid- $\mathrm{d}_{2}$ and isophthalic acid- $\mathrm{d}_{2}$.

As observed in the spectra of benzoic acid- $\mathrm{d}_{1}$ and phthalic acid- $\mathrm{d}_{2}$, loss of $\cdot \mathrm{OD}$ can occur without the equilibration process. This is illustrated by the fact that the peaks at masses 150 and 151 and the metastable peaks at masses 99.2 and 100.2 arising from them are not in the predicted ratios from equilibration considerations.

In the cases of the deuterated terephthalic and isophthalic acids there was some difficulty in estimating the relative areas of the meta-stable peaks concerned because the samples were not completely deuterated. The undeuterated species give rise to a meta-stable peak at 133.7 due to the transition $166^{+} \rightarrow 149^{+}+{ }^{\circ} \mathrm{OH}$, which interferes with the peak at 133.9 due to loss of $\cdot \mathrm{OD}$ from the dideutero species. The "true" height of the peak at 133.9 was estimated by subtraction from its apparent height of the appropriately normalised peak from the spectrum of pure undeuterated material. Although this correction decreases the accuracy of determination of the peak heights, the measured ratios of the metastable peaks for loss of $\cdot \mathrm{OH}$ and $\cdot \mathrm{OD}$ from the parent ion in the spectra of terephthalic acid- $\mathrm{d}_{2}$ and isophthalic acid- $\mathrm{d}_{2}$ were close to the predicted values of $2: 1$ and $3: 2$ respectively.

The spectra of the four undeuterated acids are illustrated in Fig. 8. (a)

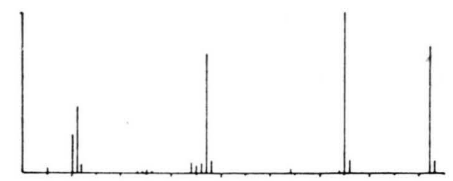

(b)

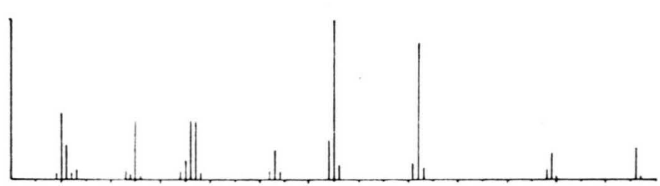

(c)

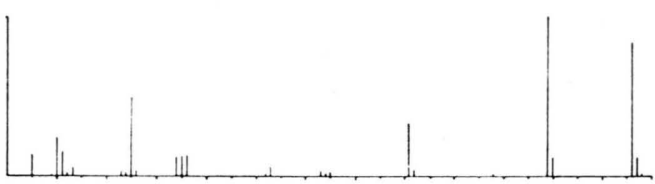

(d)

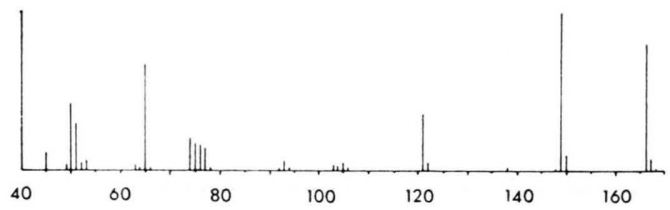

Fig. 8. The mass spectra of benzoic acid (a), phthalic acid (b), isophthalic acid (c), and terephthalic acid (d).

\section{Conclusions}

The fragmentation processes in the mass spectra of the simple aromatic carboxylic acids can be explained in terms of simple decompositions involving the loss of small neutral fragments. The large majority of the steps are verified by the observation of meta-stable peaks.

When these acids contain deuterium on the carboxylic acid groups, exchange of the deuterium atom with hydrogen atoms ortho to the acid group can occur in some of the ion species. This is observed when rotation of the acid group about the bond joining it to the aromatic system is unrestricted.

Note added in proof: The authors would like to acknowledge a similar study with which they have recently been acquainted: S. Meyerson and J. L. Corbin, J. Amer. Chem. Soc., in press. 